\title{
Simultaneous Breast Expression in Breastfeeding Women Is More Efficacious Than Sequential Breast Expression
}

\author{
Danielle K. Prime, Catherine P. Garbin, Peter E. Hartmann, and Jacqueline C. Kent
}

\begin{abstract}
Introduction: Simultaneous (SIM) breast expression saves mothers time compared with sequential (SEQ) expression, but it remains unclear whether the two methods differ in milk output efficiency and efficacy.

Subjects and Methods: The Showmilk device (Medela AG, Baar, Switzerland) was used to measure milk output and milk ejection during breast expression (electric pump) in 31 Australian breastfeeding mothers of term infants (median age, 19 weeks [interquartile range, 10-33 weeks]). The order of expression type (SIM/SEQ) and breast (left/right) was randomized.

Results: SIM expression yielded more milk ejections $(p \leq 0.001)$ and greater amounts of milk at 2,5 , and 10 minutes $(p \leq 0.01)$ and removed a greater total amount of milk $(p \leq 0.01)$ and percentage of available milk $(p<0.05)$ than SEQ expression. After SIM expression the cream content of both the overall $(8.3 \%$ [ $p \leq 0.05])$ and postexpression $(12.6 \%$ [ $p \leq 0.001])$ milk were greater. During SEQ expression, the breast expressed first had a shorter time to $50 \%$ and $80 \%$ of the total amount of milk than the breast expressed second ( $p \leq 0.05)$, but, overall, a similar percentage of available milk was removed from both breasts.

Conclusions: SIM expression stimulated more milk ejections and was a more efficient and efficacious method of expression, yielding milk with a higher energy content.
\end{abstract}

\section{Introduction}

I N DEVELOPED COUNTRIES a large proportion of breastfeeding mothers are now using breast pumps to express their milk for health issues or convenience. ${ }^{1}$ Additionally, concerns about low milk supply are cited as the most common reason for breastfeeding difficulties, ${ }^{2}$ and expressing both breasts postbreastfeeds is the recognized management for increasing milk supply when required. ${ }^{3}$ Therefore, mothers need access to (1) breast pumps that are efficient (minimizing time and effort) and efficacious (maximizing milk removal) and (2) evidence-based information on how to best express milk with a breast pump. This will allow mothers to make informed decisions when choosing the method of breast expression that is best suited to their situation.

If mothers choose to use an electric breast pump, then they may have the option of expressing milk using the simultaneous (SIM) (both breasts at the same time) or the sequential (SEQ) (one breast followed by the other) technique. Researchers have attempted to understand whether these techniques differ in efficiency and efficacy, with four studies investigating mothers of premature infants ${ }^{4-7}$ and two studies investigating mothers of healthy term infants. ${ }^{8,9}$
Comparing these studies is difficult as the milk expression protocols differ with respect to the duration of each expression session. In addition, the measured outcome variables varied from total milk yield at a single expression session to long-term daily or weekly milk productions. However, all the studies found that SIM saved time for mothers. In some cases this was demonstrated by a reduction in the number of hours spent expressing or by mothers' preference for SIM due to the reduction in time associated with it. Although all studies recommended SIM to be the preferred technique, only one study in each of these populations of women demonstrated a significant increase in milk yield during SIM. ${ }^{7,8}$

Previously, efficacy of milk expression has been assessed by overall milk output. However, because mothers and infants vary greatly in their feeding patterns, milk production, and breastmilk storage capacity, ${ }^{10}$ the total volume expressed for one mother can be very different from that of another, yet both mothers may have removed a similar proportion of the milk contained in their breasts.

Technology is now available that measures milk flow from the breast throughout breast expression. ${ }^{11}$ This allows measurement of milk removal efficiency as well as the number of milk ejections occurring during each breast expression session. Furthermore, the efficacy of milk removal can be

School of Chemistry and Biochemistry, The University of Western Australia, Crawley, Western Australia, Australia. 
measured by not only the total volume of milk expressed, but also by calculating for each mother the amount of milk available in the breast prior to expression and what proportion of that milk is removed by the expression.

The aim of this study was to investigate with modern technology whether milk removal with an electric breast pump was significantly different during SIM compared with SEQ expression. Our outcome measures included milk removal efficiency, efficacy, number of milk ejections, and milk cream content. Furthermore, we aimed to determine whether any differences existed during SEQ expression between the breast that was expressed first compared with the breast that was expressed second.

\section{Subjects and Methods}

\section{Subjects}

Healthy Australian breastmilk-feeding mothers $(n=31)$ of term infants were recruited through local Community Health Nurses, the Australian Breastfeeding Association (Western Australia Branch), or advertisements on the Web site of The University of Western Australia. To be eligible for participation, mothers needed to have an established milk supply and have no concerns about their milk production. The mothers in this study were exclusively breastfeeding, breastfeeding and expressing, or exclusively expressing breastmilk. All mothers supplied written, informed consent to participate in the study, which was approved by the Human Research Ethics Committee at The University of Western Australia.

The mothers attended The University of Western Australia research room at the Breast Feeding Centre of Western Australia at King Edward Memorial Hospital for Women (Subiaco, Perth, WA, Australia) for two study sessions occurring within 5 weeks of each other. During one session both breasts were expressed simultaneously (SIM technique), and during the other session the breasts were expressed sequentially (SEQ technique). The order of SIM or SEQ was randomized by allocating SIM on the first visit for every alternate mother as they were enrolled, and the order of the breast that was expressed first during SEQ was randomized by allocating the left breast as the first to be expressed for every alternate SEQ session. There was no prescribed interval since the previous breastfeed or expression before the study session.

\section{Breast expression}

An electric breast pump (Symphony ${ }^{\circledR}$; Medela AG, Baar, Switzerland) and standard 24-mm breastshields were used. The stimulation pattern (120 cycles per minute) was applied by the pump at a vacuum chosen by the mother until milk ejection was detected by the mother's sensation or a marked increase in milk flow was noted or for a maximum of 2 minutes. The pump was then changed to the expression pattern (54-78 cycles per minute), and the vacuum was adjusted to the mother's maximum comfortable vacuum, for each breast. Expression continued for 15 minutes after the first milk flow was recorded, both during SIM and for each breast during SEQ. All equipment in contact with breastmilk was sterilized before use, and the expressed milk was returned to the mother.

As described previously ${ }^{11}$ milk was conveyed via a connecting tube from the breastshield to one of three bottles placed on the weigh platform of a continuous weigh balance
(Showmilk; Medela AG). The length of the connecting tube was adjusted to remove loops and avoid pooling to ensure unimpeded flow of milk from the breastshield to the bottles on the balance. The first $1 \mathrm{~mL}$ (first milk) was collected into the first bottle, the bulk of the milk was collected into the second bottle (expressed milk), and the last milk expressed was collected into the third bottle (last milk).

The Showmilk device measures the cumulative weight of milk at $50 \mathrm{~Hz}$ with a resolution of $0.1 \mathrm{~g}$ and accuracy $\pm 0.02 \%$ to a maximum of $2 \mathrm{~kg}$. It also has two analog sensor inputs that were used to measure breast pump vacuum. The Showmilk was connected to a computer using a USB interface that included interactive software (Showmilk version 1.3.1.2, 20072008; (C) Medela AG) to allow real-time visualization of not only the cumulative weight of milk but also the derivative, which is the flow rate of the milk. Recorded data were deidentified, saved, and exported. Using a purpose-built macro (Carag AG, Baar), the data were then imported into a spreadsheet (Microsoft ${ }^{\circledR}$ Office Excel 2010; Microsoft, Redmond, WA) for analysis. Cumulative weight measurements were sampled at $10 \mathrm{~Hz}$, and the derivative (rate of milk flow, in $\mathrm{g}$ /second) was calculated and graphed. Peaks in flow rate that have a steep incline followed by a slower decline typify a milk ejection, ${ }^{11}$ and thus milk ejections can be quantified visually. This typical shape helps to differentiate a physiological flow rate increase resulting from a milk ejection from an artifact such as pooling of milk in the shield that is released intermittently.

\section{Measurement of 24-hour milk production and calculation of storage capacity of the breast}

Milk production was measured by the mothers over a 24 28-hour period of breastfeeding and/or expressing in their own homes at their convenience within 2 weeks of the first and last study sessions. Mothers test-weighed their infants before and after each breastfeed, or weighed the bottle before and after each expression, from each breast. ${ }^{12}$ During this period, mothers hand-expressed small milk samples $(<1 \mathrm{~mL})$ into 5-mL polypropylene vials (Disposable Products, Adelaide, Australia), immediately before and after each breastfeed or expression. The breastfeed volumes and cream contents of the milk samples (measured by the creamatocrit method $^{13}$ on the Creamatocrit Plus ${ }^{\mathrm{TM}}$ device ${ }^{14}$ [Medela Inc., McHenry, IL]) were used to calculate the breastfeeding storage capacity as described previously. ${ }^{15}$ Before and after each pumping session the mothers hand-expressed small milk samples $(<1 \mathrm{~mL})$ from the breast being expressed. The cream content of these milk samples and volume of the expressions, combined with the data from the 24-hour period, were used to calculate the potential storage capacity of the breast as described previously, ${ }^{15}$ and the cream content of the initial milk sample was used to calculate the degree of fullness of the breast at the beginning of the pumping session. ${ }^{15}$ Multiplication of the degree of fullness by the potential storage capacity gave the amount of milk available in the breast before the experimental expression sessions. ${ }^{15}$ The amount of milk expressed as a percentage of the amount of milk available gave a measure of the efficacy of the expression session.

When a mother chose not to measure her milk production over a 24-hour period, the cream content of the milk samples expressed before and after each study session and the 
volumes expressed during those study sessions were used to calculate the potential storage capacity of the breasts.

\section{Statistical analysis}

All analyses were performed using $\mathrm{R}$ version 2.10.1 for Windows ${ }^{16}$ and the package nlme. ${ }^{17}$ Data exploration consisted of box plots and descriptive statistics. Milk production between breasts was compared using paired Student's $t$ tests. Effect of expression type (SIM vs. SEQ) on the expression variables was assessed using linear mixed-effects models, with fixed effects of expression type, feed order (breast expressed first vs. second), and breast (left vs. right) and random effects of different baseline levels for each participant. Appropriateness of models was assessed visually from residual plots. Summary statistics are presented as mean $\pm S D$ values unless otherwise stated. Differences were considered to be significant at $p<0.05$.

\section{Results}

Table 1 summarizes the demographic data of 30 of the 31 fully or partially breastfeeding mothers (one mother did not complete the questionnaire). Table 1 also includes the milk production characteristics of the 25 mothers completing their 24-hour milk production measurements (six mothers did not complete this measurement). The potential breast storage capacity (based on the expression data) was calculated for all mothers $(n=31)$. There were equal numbers of male and female infants, and the majority of mothers were primiparous $(70 \%)$. There was no significant difference between the left and right breasts in milk production, average breastfeed or expression amount, or breastfeeding or potential storage capacity. The potential storage capacity was greater than the breastfeeding storage capacity for some mothers, and there was an overall difference $(p<0.05)$ (Table 1$)$.

\section{SIM compared with SEQ expression}

The expression characteristics for SIM and SEQ are shown in Table 2. Prior to breast expression, mothers had a similar

Table 1. Demographics and Milk Production Characteristics

\begin{tabular}{lc}
\hline & $\begin{array}{c}\text { Median (interquartile } \\
\text { range) }\end{array}$ \\
\hline Maternal age (years) & $31.8(29.9-35.5)$ \\
Maternal body mass index $\left(\mathrm{kg} / \mathrm{m}^{2}\right)$ & $23.5(21.6-26.5)$ \\
Parity & $1.0(1.0-2.0)$ \\
Infant gestational age (weeks) & $40.0(39.0-40.2)$ \\
Infant birth weight $(\mathrm{g})$ & $3,470(3,310-3,805)$ \\
Total milk production (g/24 hours) & $788(611-890)$ \\
$\quad$ Left breast & $407(307-449)$ \\
$\quad$ Right breast & $380(301-461)$ \\
Average breastfeed or expression $(\mathrm{g})$ & $71(57-80)$ \\
$\quad$ Left breast & $63(48-81)$ \\
$\quad$ Right breast & \\
Breastfeeding storage capacity (mL) & $143(117-194)$ \\
$\quad$ Left breast & $167(123-195)$ \\
Right breast & $159(113-194)$ \\
Potential storage capacity (mL) & $163(105-222)$ \\
$\quad$ Reft breast & \\
\hline
\end{tabular}

Table 2. Milk Expression Characteristics During the Simultaneous and Sequential Methods OF BREAST EXPRESSION

\begin{tabular}{lcc}
\hline & Simultaneous & Sequential \\
\hline Time to first recorded milk & $102 \pm 71$ & $111 \pm 84$ \\
$\quad$ flow (seconds) & & \\
Milk yield (g) at & $31 \pm 23$ & $23 \pm 20^{\mathrm{b}}$ \\
2 minutes & $58 \pm 38$ & $45 \pm 37^{\mathrm{a}}$ \\
5 minutes & $76 \pm 44$ & $58 \pm 43^{\mathrm{b}}$ \\
10 minutes & $82 \pm 51$ & $70 \pm 53^{\mathrm{b}}$ \\
Total milk yield (g) at 15 & & \\
$\quad$ minutes & $39 \pm 17$ & $38 \pm 20$ \\
Percentage total milk yield at & & $69 \pm 20$ \\
2 minutes & $93 \pm 7$ & $88 \pm 12^{\mathrm{b}}$ \\
5 minutes & $170 \pm 103$ & $200 \pm 142$ \\
$\quad$ 10 minutes & & \\
Time (seconds) to 50\% & & \\
$\quad$ of total milk yield & $340 \pm 162$ & $387 \pm 178$ \\
Time (seconds) to 80\% & & \\
$\quad$ of total milk yield & $0.69 \pm 0.28$ & $0.63 \pm 0.26$ \\
Initial degree of fullness & $119 \pm 85$ & $114 \pm 87$ \\
Available milk (g) & & \\
Cream (\%) in & $4.4 \pm 2.7$ & $4.6 \pm 2.7$ \\
$\quad$ First milk & $8.3 \pm 2.8$ & $7.3 \pm 2.6^{\mathrm{a}}$ \\
Expressed milk & $12.6 \pm 4.4$ & $10.5 \pm 4.1^{\mathrm{c}}$ \\
Last milk & & \\
Final degree of fullness & $0.03(0.00-0.18)$ & $0.16(0.06-0.29)^{\mathrm{a}}$ \\
Number of milk ejections & $4.4 \pm 1.7$ & $3.4 \pm 1.4^{\mathrm{c}}$ \\
\hline
\end{tabular}

Data are mean $\pm S D$ values or median (interquartile range).

Significant differences between the two expression methods are indicated: ${ }^{\mathrm{a}} p<0.05,{ }^{\mathrm{b}} p<0.01,{ }^{\mathrm{c}} p<0.001$.

initial degree of breast fullness, amount of milk available in the breasts, and initial cream content on the two experimental days. No significant difference was found between the two expression techniques in the time to first recorded milk flow (first milk ejection) (Table 2). In addition, the stimulation and expression vacuums selected by the mothers were similar for the two methods of expression.

Significant differences were observed between the two expression techniques in their milk output (Table 2). SIM produced a significantly higher yield of milk at the 2-, 5-, 10-, and 15minute time points. The percentage of the total milk yield was significantly higher using SIM after 10 minutes of expression.

The percentage of available milk removed after 5, 10, and 15 minutes was significantly higher using SIM compared with SEQ (Fig. 1), but the shorter time taken to remove $50 \%$ and $80 \%$ of the total milk yield did not reach significance (Table 2 ).

The number of milk ejections was significantly higher using SIM than using SEQ. The timing or location of the additional milk ejection(s) did not occur with any consistency in the mother's milk ejection pattern.

The final degree of breast fullness was significantly lower after SIM compared with SEQ. Furthermore, the last drops of milk during SIM contained a significantly higher cream content than during SEQ, and the overall cream content of the total expressed milk was also significantly higher after SIM.

\section{Left and right breasts during expression}

There were no significant differences between the left and right breasts in any of the measured parameters when the 


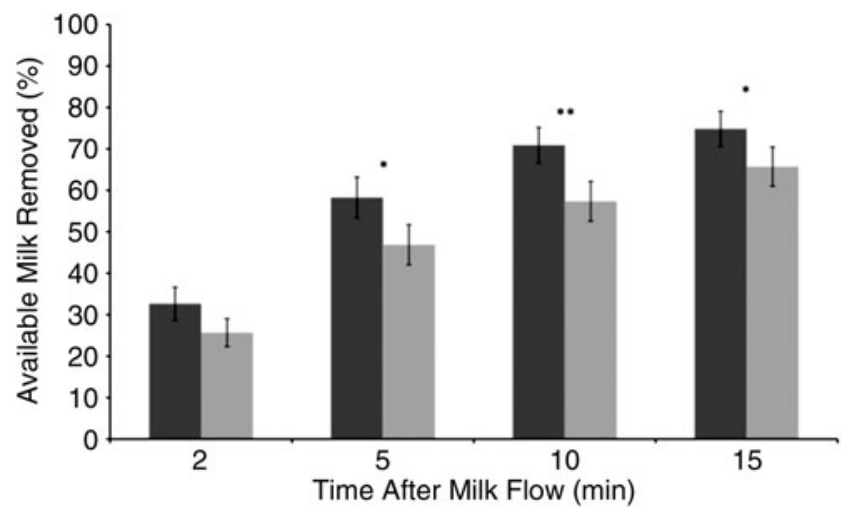

FIG. 1. Percentage of available milk removed after 2, 5, 10, and 15 minutes of expression during use of the simultaneous (dark gray) and sequential (light gray) techniques $(n=31)$. Significant differences were identified using linear mixed effects modeling and are indicated: ${ }^{*} p<0.05,{ }^{* *} p<0.01$.

breasts were expressed simultaneously (SIM data only). When comparing all breasts (SIM and SEQ data combined) for left and right breast differences, the only significant difference in milk output measurements was the milk yield after 5 minutes of expression (left, $46 \pm 31 \mathrm{~g}$; right, $57 \pm 44 \mathrm{~g}[p<0.05]$ ).

When only analyzing the SEQ data, significant differences were found between the left and right breasts in the time since last breastfeed (left, $2.7 \pm 1.4$ hours; right, $3.3 \pm 1.4$ hours $[p<0.05]$ ), the number of milk ejections (left, 3.7 \pm 1.4 ; right, $3.1 \pm 1.4[p<0.05]$ ), the milk yield at 5 minutes (left, $37 \pm 25 \mathrm{~g}$; right, $53 \pm 45 \mathrm{~g}[p<0.05])$, and the percentage of total milk yield at 5 minutes (left, $63 \pm 21 \%$; right, $75 \pm 18 \%[p<0.05]$ ).

Differences between the left and right breasts were accounted for in the modeling used to compare the two expression methods and order of sequential breasts. All significant differences reported remained significant regardless of these breast differences.

\section{Breast order during sequential expression}

The expression characteristics for the breast expressed first and second are shown in Table 3 . There was a difference between the first and second breast for SEQ in the initial degree of fullness of the breast, yet no difference was observed in the time since last breastfeed for the breast expressed first (3.1 \pm 1.4 hours) and second ( $2.9 \pm 1.4$ hours). Furthermore, there was a difference $(p<0.01)$ between the vacuum chosen for the stimulation pattern between the breast expressed first $(-102 \pm 34 \mathrm{~mm} \mathrm{Hg})$ and second $(-123 \pm 46 \mathrm{~mm} \mathrm{Hg})$; however, no significant difference was observed for the expression vacuum chosen for the breast expressed first $(-188 \pm 60 \mathrm{~mm}$ $\mathrm{Hg})$ and second ( $-202 \pm 52 \mathrm{~mm} \mathrm{Hg}$ ).

There were significant differences in the expression characteristics between the breasts according to the order in which they were expressed (first/second) that were not affected by differences between the left and right breast or by differing initial degrees of breast fullness. The percentage of total milk yield removed at 5 and 10 minutes was higher in the first breast, and the time to reach $50 \%$ and $80 \%$ of the total milk yield was shorter for the first breast expressed (Table 3). No differences were observed in the total milk yield, the percentage of available milk removed at any time point, the
Table 3. Milk Expression Characteristics FOR THE BREAST EXPRESSEd First AND SECOND Using the Sequential Method of Breast Expression

\begin{tabular}{|c|c|c|}
\hline & First & Second \\
\hline $\begin{array}{l}\text { Time to first recorded milk } \\
\text { flow (seconds) }\end{array}$ & $116 \pm 75$ & $109 \pm 92$ \\
\hline \multicolumn{3}{|l|}{ Milk yield $(\mathrm{g})$ at } \\
\hline 2 minutes & $26 \pm 23$ & $20 \pm 17$ \\
\hline 5 minutes & $51 \pm 41$ & $39 \pm 33$ \\
\hline 10 minutes & $63 \pm 48$ & $54 \pm 38$ \\
\hline Total milk yield (g) at $15 \mathrm{~min}$ & $74 \pm 60$ & $65 \pm 46$ \\
\hline \multicolumn{3}{|l|}{ Percentage total milk yield at } \\
\hline 2 minutes & $40 \pm 19$ & $35 \pm 22$ \\
\hline 5 minutes & $75 \pm 17$ & $62 \pm 22^{b}$ \\
\hline 10 minutes & $92 \pm 7$ & $85 \pm 14^{\mathrm{a}}$ \\
\hline $\begin{array}{l}\text { Time (seconds) to } 50 \% \\
\text { of total milk yield }\end{array}$ & $153 \pm 85$ & $246 \pm 170^{b}$ \\
\hline $\begin{array}{l}\text { Time (seconds) to } 80 \% \\
\text { of total milk yield }\end{array}$ & $338 \pm 145$ & $437 \pm 197^{\mathrm{a}}$ \\
\hline Initial degree of fullness & $0.71 \pm 0.27$ & $0.56 \pm 0.23$ \\
\hline Available milk (g) & $128 \pm 98$ & $100 \pm 73$ \\
\hline $\begin{array}{l}\text { Percentage available } \\
\text { milk at } 15 \text { minutes }\end{array}$ & $62 \pm 25$ & $70 \pm 28$ \\
\hline \multicolumn{3}{|l|}{ Cream $(\%)$ in } \\
\hline First milk & $4.1 \pm 3.0$ & $5.1 \pm 2.3$ \\
\hline Expressed milk & $7.4 \pm 3.0$ & $7.2 \pm 2.3$ \\
\hline Last milk & $10.1 \pm 4.5$ & $10.8 \pm 3.7$ \\
\hline \multirow[t]{2}{*}{ Final degree of fullness } & 0.25 & 0.17 \\
\hline & $(0.08-0.34)$ & $(0.04-0.24)$ \\
\hline Number of milk ejections & $3.3 \pm 1.5$ & $3.5 \pm 1.4$ \\
\hline
\end{tabular}

Results are mean \pm SD values or median (interquartile range).

Significant differences between the first and second breasts are indicated: ${ }^{\mathrm{a}} p<0.05,{ }^{\mathrm{b}} p<0.01$.

cream content of the last drops of milk, or the final degree of breast fullness.

\section{Discussion}

Our results confirm a significant benefit for mothers in both efficiency and efficacy of breast expression using the SIM compared with the SEQ technique when using an electric breast pump. Furthermore, we were able to identify a potential mechanism for this advantage: an increase in the measured number of milk ejections (Table 2). Moreover, the improved efficacy of SIM expression resulted in milk that had a higher cream content compared with SEQ expression (Table 2). Given the close relationship between the cream content and the energy content of milk, ${ }^{14}$ the milk yielded by SIM will have a higher energy content than that yielded by SEQ.

The efficiency of breast expression can be assessed by measuring, at intervals during expression, the milk yield, the percentage of total milk yield, and the percentage of available milk removed (higher is more efficient) and/or the time taken to reach $50 \%$ and $80 \%$ of the total milk yield (shorter is more efficient). SIM improved the efficiency of breast expression, shown by a higher milk yield at 2, 5, and 10 minutes, a higher percentage of total milk yield at 10 minutes (Table 2), and a higher percentage of available milk removed at 5, 10, and 15 minutes after first milk ejection (Fig. 1). The time taken to remove $50 \%$ and $80 \%$ of the total milk yield using SIM tended 
to be shorter than SEQ, although this did not reach statistical significance ( $p=0.129$ and $p=0.095$, respectively). In addition, using SIM saves the mother time compared with using SEQ, as noted in previous studies. ${ }^{4,6-8,18}$

The efficacy of breast expression can be assessed by measuring the total milk yield or, preferably, the percentage of available milk removed. This controls for mothers having vastly different milk productions ${ }^{10}$ and for each mother having a different degree of fullness before each pumping session. In addition, measurement of the cream content of the last milk expressed indicates how well the breast has been drained. ${ }^{19}$

SIM expression for 15 minutes yielded more milk than SEQ expression of each breast for 15 minutes. This supports the results of Jones et al., ${ }^{7}$ who showed a significantly higher yield of milk after SIM compared with SEQ when the duration of expression was not limited. We also showed that more milk was removed after 5 minutes during SIM compared than SEQ, similar to the results of Auerbach. ${ }^{8}$ The lack of consistency in the results of previous studies is likely due to the output measures used to indicate efficacy. These studies recorded the mean weekly or daily milk output of mothers of premature infants $^{4-6}$ and found trends for milk yield to be higher for mothers using SIM, but the differences were not significant.

Our study showed that the percentage of available milk removed was higher using SIM. In fact, $75 \%$ of the available milk was removed during SIM, compared with $66 \%$ during SEQ (Fig. 1). This percentage for SIM is also higher than that measured in previous studies in which only one breast was pumped. ${ }^{15,20}$ Studies also show the healthy breastfeeding baby on average removes $67 \%$ of the available milk. ${ }^{10}$ This highlights that SIM will increase breast drainage and stimulate a higher rate of milk synthesis, ${ }^{21}$ giving the mother a greater chance of increasing her milk supply. In addition, we found that the last milk collected from SIM was of a higher cream content than the last milk collected from SEQ, further supporting superior drainage of the breast.

A higher number of milk ejections was observed using SIM, yet the time until the first milk ejection occurred was not different. This implies that stimulation of both nipples at the same time does not lead to a shorter time to first milk ejection, but does stimulate the mother to have an extra milk ejection during the 15-minute expression period. For the first time, this finding allows us to understand a possible mechanism for the increased efficiency and efficacy measured with SIM. The basis for this mechanism can also be supported by previous findings that the amount of milk removed from the breast by a breastfeeding infant is related to the number of milk ejections, irrespective of the time taken for the breastfeed. ${ }^{22}$ Previous studies have investigated differences in prolactin levels between SIM and SEQ as a possible mechanism for increased milk production. Results remain inconclusive, with one study finding an increase in prolactin, ${ }^{9}$ and others finding no difference. ${ }^{4}$ However, it has been shown that although prolactin is required for milk production, increased concentrations are not associated with increased milk production. ${ }^{23}$

This is the first time that the pattern of milk ejections during breast expression has been shown to differ within a mother. Previous research has demonstrated that mothers have a repeatable pattern of milk ejection at repeated simultaneous breast expression sessions from 1 to 9 months of lactation ${ }^{24}$ and that this pattern does not change with differing breast- shield sizes. ${ }^{25}$ The current study showed that the pattern of milk ejections does differ with expression methods. Mothers had a different number of milk ejections between SIM and SEQ without reportable consistency in the timing of the milk ejections, with some changes occurring early in the session and some later. Further investigation is required to characterize the differences in the milk ejection patterns observed.

To further investigate reasons why SEQ was less efficacious, we compared the breast that was expressed first during SEQ with the breast that was expressed second. For the breast expressed first there was a shorter time to reach $50 \%$ and $80 \%$ of the total milk yield and a higher milk yield at 5 and 10 minutes than the breast expressed second (Table 3). However, no differences were observed in any measures of overall milk yield and efficacy, indicating that expression from the first breast was significantly more efficient compared with the second breast, but the efficacy after 15 minutes was similar.

The reasons for the difference between the efficiency of the first and second breasts have not been determined; however, it can be speculated that after experiencing exposure to, and response to, systemic oxytocin for 15 minutes, the response of the breast expressed second is diminished during the early minutes of expression. Because it is known that the first two milk ejections contribute the largest proportion of milk, ${ }^{15,26}$ this diminished response would have a significant effect on efficiency of expression. However, when pumping was continued for a total of 15 minutes, the breast expressed second reached the same total milk yield. Our results indicate that although expressing for only 10 minutes from the first breast removes more than $90 \%$ of the milk that will be yielded from that breast, longer expression durations are required to reach the same milk output for the second breast. It can also be speculated that including a break between expressing the first and second breasts may eliminate the lower efficiency measured in this study, but this remains to be investigated. During breastfeeding, infants taking paired breastfeedings feed from the breasts sequentially (unless the mother is simultaneously feeding twins). When breastfeedings are paired, the amount of milk removed from the second breast by the infant is the same as the first breast if the second breast is the more productive breast, and less if the second breast is the less productive breast. ${ }^{10}$ This may be a result not only of the infant's appetite but also of the slower release of milk from the second breast.

Differences between the left and right breasts in the milk yield and percentage of total milk yield after 5 minutes of expression were found during SEQ even though neither the degree of breast fullness nor the amount of available milk differed between the breasts. Differences between the left and right breasts of individual mothers are common for overall milk outputs during expression. ${ }^{27,28}$ Anecdotally, many mothers will indicate that their baby prefers a particular breast, and, indeed, differences between left and right breasts have been observed during breastfeeding ${ }^{10}$ and in short-term rates of milk synthesis. ${ }^{23}$ These differences between the breasts were not observed during SIM, indicating that SIM expression improves the efficiency of expression of the breast that was less efficient during SEQ. These results indicate that mothers should opt for SIM expression where possible, not only for the advantages of milk yield and time saving, but also for the advantage of optimizing milk removal from both breasts. Furthermore, the results imply that breastfeeding 
twins simultaneously may be more advantageous than feeding the two infants sequentially.

\section{Conclusions}

In term mothers SIM expression stimulated more milk ejections and was a more efficient and efficacious method of breast expression, yielding milk with a higher energy content. During SEQ expression, the first breast was more efficient than the second breast, but milk removal from the second breast was equally efficacious after 15 minutes. This study has demonstrated increased efficiency and efficacy with the SIM technique and also identified milk ejection as a possible physiological mechanism. Further studies are required to confirm that these findings will also assist expressing mothers of preterm infants.

\section{Acknowledgments}

We thank the mothers and infants participating in the study, the Australian Breastfeeding Association, and the Child and Adolescent Community Health Nurses in the Oceanic Health Region. The authors gratefully acknowledge statistical advice from Anna Hepworth. The study was funded by the Women and Infants Research Foundation and an unrestricted research grant from Medela AG (Switzerland).

\section{Disclosure Statement}

No competing financial interests exist.

\section{References}

1. Binns C, Win N, Zhao Y, et al. Trends in the expression of breastmilk 1993-2003. Breastfeed Rev 2006;14:5-9.

2. Amir LH. Breastfeeding-Managing 'supply' difficulties. Aust Fam Physician 2006;35:686-689.

3. Kent JC, Prime DK, Garbin C. Principles for maintaining or increasing breast milk production. J Obstet Gynecol Neonatal Nurs 2012;41:114-121.

4. Groh-Wargo S, Toth A, Mahoney K, et al. The utility of a bilateral breast pumping system for mothers of premature infants. Neonatal Netw 1995;14:31-36.

5. Hill PD, Aldag JC, Chatterton RT. The effect of sequential and simultaneous breast pumping on milk volume and prolactin levels: A pilot study. J Hum Lact 1996;12:193-199.

6. Hill PD, Aldag JC, Chatterton RT. Effects of pumping style on milk production in mothers of non-nursing preterm infants. J Hum Lact 1999;15:209-216.

7. Jones E, Dimmock PW, Spencer SA. A randomised controlled trial to compare methods of milk expression after preterm delivery. Arch Dis Child Fetal Neonatal Ed 2001;85: F91-F95.

8. Auerbach KG. Sequential and simultaneous breast pumping: A comparison. Int J Nurs Stud 1990;27:257-265.

9. Neifert M, Seacat J. Milk yield and prolactin rise with simultaneous breast pumping. Presented at the Ambulatory Pediatric Association Annual Meeting, Washington, DC, 1985.

10. Kent JC, Mitoulas LR, Cregan MD, et al. Volume and frequency of breastfeeds and fat content of breastmilk throughout the day. Pediatrics 2006;117:e387-e395.

11. Prime DK, Geddes DT, Spatz DL, et al. Using milk flow rate to investigate milk ejection in the left and right breasts during simultaneous breast expression in women. Int Breastfeed J 2009;4:10.

12. Arthur PG, Hartmann PE, Smith M. Measurement of the milk intake of breast-fed infants. J Pediatr Gastroenterol Nutr 1987;6:758-763.

13. Fleet IR, Linzell JL. A rapid method of estimating fat in very small quantities of milk J Physiol (Lond) 1964;175:15-17.

14. Meier PP, Engstrom JL, Zuleger JL, et al. Accuracy of a userfriendly centrifuge for measuring creamatocrits on mothers' milk in the clinical setting. Breastfeed Med 2006;1:79-87.

15. Kent JC, Mitoulas LR, Cregan MD, et al. Importance of vacuum for breastmilk expression. Breastfeed Med 2008;3:11-19.

16. R Development Core Team. R: A Language and Environment for Statistical Computing. R Foundation for Statistical Computing, Vienna, 2007.

17. Pinheiro J, Bates D, DebRoy S, et al. Nlme: Linear and Nonlinear Mixed Effects Models. R package version 31-89. R Foundation for Statistical Computing, Vienna, 2008.

18. Fewtrell MS, Lucas P, Collier S, et al. Randomized trial comparing the efficacy of a novel manual breast pump with a standard electric breast pump in mothers who delivered preterm infants. Pediatrics 2001;107:1291-1297.

19. Kent JC. How breastfeeding works. J Midwifery Womens Health 2007;52:564-570.

20. Kent JC, Geddes DT, Hepworth AR, et al. Effect of warm breastshields on breast milk pumping. J Hum Lact 2011;27: 331-338.

21. Daly SE, Owens RA, Hartmann PE. The short-term synthesis and infant-regulated removal of milk in lactating women. Exp Physiol 1993;78:209-220.

22. Ramsay DT, Kent JC, Owens RA, et al. Ultrasound imaging of milk ejection in the breast of lactating women. Pediatrics 2004;113:361-367.

23. Cox DB, Owens RA, Hartmann PE. Blood and milk prolactin and the rate of milk synthesis in women. Exp Physiol 1996; 81:1007-1020.

24. Prime DK, Geddes DT, Hepworth AR, et al. Comparison of the patterns of milk ejection during repeated breast expression session in women. Breastfeed Med 2011;6:183-190.

25. Prime DK, Geddes DT, Spatz DL, et al. The effect of breastshield size and anatomy on milk removal in women. J Hum Lact 2011;26:433.

26. Prime DK, Kent JC, Hepworth AR, et al. Dynamics of milk removal during simultaneous breast expression in women. Breastfeed Med 2012;7:100-106.

27. Engstrom JL, Meier PP, Jegier B, et al. Comparison of milk output from the right and left breasts during simultaneous pumping in mothers of very low birthweight infants. Breastfeed Med 2007;2:83-91.

28. Hill PD, Aldag JC, Zinaman M, et al. Comparison of milk output between breasts in pump-dependent mothers. J Hum Lact 2007;23:333-337.

Address correspondence to: Danielle K. Prime, Ph.D. School of Chemistry and Biochemistry The University of Western Australia M310, 35 Stirling Highway Crawley, WA 6009, Australia

E-mail: danielle.prime@gmail.com 